\title{
Possible Relativistic Definitions of Parallax, Proper Motion and Radial Velocity
}

\section{S. Klioner}

Lohrmann Observatory, Dresden Technical University, 01062 Germany

\begin{abstract}
It is argued that the relativistic definitions of parallax, proper motion and radial velocity consistent with an accuracy of $1 \mu$ as should be considered only within a well-defined algorithm of relativistic reduction of observational data. Such an algorithm is formulated and the corresponding definitions of astrometric parameters are discussed.
\end{abstract}

\section{Introduction}

The accuracy of future space-based astrometric observations is expected to attain a level of $1 \mu$ as. In this paper we briefly describe a relativistic model of space-based optical positional observations valid at such a high-accuracy level and suggest, in particular, definitions of parallax, proper motion and radial velocity compatible with general relativity at a level of $1 \mu$ as. Although the definitions are quite simple (see Klioner and Kopeikin, 1992), their interpretation at such a high level of accuracy is rather tricky. Parallax and proper motion are no longer two independent effects. Second-order effects due to parallax and proper motion as well as the effects resulting from interaction between the two effects are important. Moreover, parallax, proper motion and other astrometric parameters are defined operationally and have some meaning only within the particular model of relativistic reductions chosen. That is why the whole relativistic model of observations must be considered. It is also clear that in order to convert observed proper motion and radial velocity into true tangential and radial velocities of the observed object, additional information is required. Since that information is not always available, the concepts of "apparent proper motion", "apparent tangential velocity" and "apparent radial velocity" are suggested. These concepts represent useful information about the observed object and should be distinguished from "true tangential velocity" and "true radial velocity". Definitions of all these concepts are discussed below.

Throughout this paper the following constraints on the various parameters are used to decide if a particular effect should be retained to attain the accuracy of $1 \mu$ as: (1) barycentric position of the observer: $\left|\mathbf{x}_{s}\right| \leq 1$ a.u. $+2 \cdot 10^{6} \mathrm{~km} ;(2)$ barycentric velocity of the observer: $\left|\dot{\mathbf{x}}_{s}\right| \leq 4 \cdot 10^{4} \mathrm{~m} / \mathrm{s}$; (3) parallax $\pi \leq 1^{\prime \prime}$.

\section{Relativistic model of positional observations}

A relativistic model for optical positional observations having an accuracy of 1 $\mu$ as is outlined below. It is assumed that the observations are performed from 
an Earth satellite or a space station whose position $\mathbf{x}_{s}$ relative to the Barycentric Reference System (BRS) of the solar system is known for any moment of barycentric coordinate time $t \equiv T C B$. The relativistic model to be discussed below consists of several subsequent steps accounting for the following effects:

(1) aberration (effects vanishing together with the barycentric velocity of the observer). This step converts the observed direction to the source $\mathbf{s}$ into the unit BRS coordinate velocity of the light ray $\mathbf{n}$ at the point of observation $\mathbf{x}_{\boldsymbol{s}}$;

(2) gravitational light deflection for the source at infinity. This step converts $\mathbf{n}$ into the unit direction of propagation $\boldsymbol{\sigma}$ of the light ray infinitely far from the solar system;

(3) coupling of finite distance to the source and the gravitational light deflection in the gravitational field of the solar system. This step converts $\boldsymbol{\sigma}$ into a unit coordinate BRS direction $\mathbf{k}$ going from the source to the observer;

(4) parallax. This step converts $\mathbf{k}$ into a unit BRS direction $\mathbf{l}$ going from the barycenter of the solar system to the source;

(5) proper motion. This step provides a reasonable parameterization of the time dependence of 1 caused by the motion of the source relative to the BRS.

\subsection{Aberration}

The first step is to get rid of the aberrational effects related to the BRS velocity of the observer. Let $\mathbf{s}$ denote the unit direction toward the source as observed by the observer. Let $\mathbf{p}$ be the BRS coordinate velocity of the photon in the point of observation. ( $\mathbf{p}$ is directed from the source to the observer.) The unit BRS coordinate direction of the light ray $\mathbf{n}=\mathbf{p} /|\mathbf{p}|$ can be computed as

$$
\mathbf{s}=-\mathbf{n}+\frac{1}{c} \mathbf{f}_{1}+\frac{1}{c^{2}} \mathbf{f}_{2}+\frac{1}{c^{3}} \mathbf{f}_{3}+\mathcal{O}\left(c^{-4}\right) \text {. }
$$

This formula contains relativistic aberrational effects $\mathbf{f}_{i}=\mathbf{f}_{i}\left(\mathbf{n}, \dot{\mathbf{x}}_{\boldsymbol{s}}\right)$ up to thirdorder in $1 / c$. Explicit form of $\mathbf{f}_{i}$ can be found in (Klioner, 1991; Klioner, Kopeikin, 1992). Because of the first-order aberrational terms (classical aberration) the BRS coordinate velocity of the satellite must be known to an accuracy of $10^{-3} \mathrm{~m} / \mathrm{s}$ in order to attain an accuracy of $1 \mu$ as. For a satellite with the BRS velocity $\left|\dot{\mathbf{x}}_{s}\right|<40 \mathrm{~km} / \mathrm{s}$, the first-order aberration is of the order of $28^{\prime \prime}$, the second-order effect may amount to $3.6 \mathrm{mas}$, and the third-order effects are $\sim 1 \mu$ as. Note also that the higher-order aberrational effects are nonlinear with respect to the velocity of the satellite and cannot be divided into "annual" and "diurnal" aberrations as can be done with the first-order aberration for an Earth-bound observer.

\subsection{Gravitational light deflection}

The next step is to account for the general-relativistic gravitational light defection, that is to convert $\mathbf{n}$ into the corresponding unit direction $\sigma$ of the light propagation infinitely far from the gravitating sources. Relativistic equations of light propagation can be written in the form

$$
\mathbf{x}_{p}(t)=\mathbf{x}_{p}\left(t_{0}\right)+c \boldsymbol{\sigma}\left(t-t_{0}\right)+\delta \mathbf{x}_{p}(t),
$$

where $t_{0}$ is the moment of observation, $\mathbf{x}_{p}\left(t_{0}\right)$ is the position of the photon at the moment of observation (This position obviously coincides with the position of 
the satellite at that moment $\mathbf{x}_{p}\left(t_{0}\right)=\mathbf{x}_{s}\left(t_{0}\right)$.), $\boldsymbol{\sigma}$ is the unit coordinate direction of the light propagation at past null infinity

$$
\boldsymbol{\sigma}=\lim _{t \rightarrow-\infty} c^{-1} \mathbf{x}_{p}(t)
$$

and $\delta \mathbf{x}_{p}$ is the sum of all the gravitational effects in the light propagation $\left(\delta \mathbf{x}_{p}\left(t_{0}\right)=0, \lim _{t \rightarrow-\infty} \delta \dot{\mathbf{x}}_{p}(t)=0\right)$. If time dependence of the gravitational fields produced by the matter outside the solar system is negligible, those fields can be neglected while computing $\delta \mathbf{x}_{p}$. Otherwise the external fields must be taken into account (e.g., for the observations of close binary stars, where the gravitational field of the companion can cause an additional time-dependent light deflection).

The largest contribution to $\delta \mathbf{x}_{p}$ due to the solar system gravity comes from the spherically symmetric components of the gravitational fields of the massive bodies (see, e.g., Klioner and Kopeikin, 1992). Depending on the problem and the required accuracy, one can additionally consider the effects of quadrupole moments of the bodies, their rotational and translational motion as well as the post-post-Newtonian effect (Klioner, 1991a; Klioner, 1991b; Klioner and Kopeikin, 1992). It is easy to see that not only the major planets should be taken into account here, but also the Moon, Ceres and a dozen large satellites of giant planets. Detailed estimates of various effects will be published elsewhere.

Coordinate velocity of the photon can be obtained by differentiating Eq. (2): $\dot{\mathbf{x}}_{p}=c \boldsymbol{\sigma}+\delta \dot{\mathbf{x}}_{p}$ and then normalized to give the unit coordinate direction of the light propagation at the moment of observation

$$
\mathbf{n}=\boldsymbol{\sigma}+\mathbf{g}\left(\mathbf{n}, \delta \dot{\mathbf{x}}_{p}\left(t_{0}\right)\right) .
$$

\subsection{Coupling of finite distance and gravitational deflection}

The next step is to convert $\sigma$ into a BRS direction from the source to the observer. Let $\mathbf{x}_{s}(t)$ be the coordinate of the satellite at the moment of observation $t$ and $\mathbf{X}(T)$ be the position of the source at the moment of emission $T=T(t)$ of the observed signal. Let us denote

$$
\mathbf{k}(t)=\mathbf{R}(t) / R(t), \quad \mathbf{R}(t)=\mathbf{x}_{s}(t)-\mathbf{X}(T), \quad R(t)=|\mathbf{R}(t)| .
$$

Vector $\mathbf{k}$ is related to $\sigma$ as (Klioner, 1991a):

$$
\boldsymbol{\sigma}=\mathbf{k}-\mathbf{k} \times\left(\delta \dot{\mathbf{x}}_{p} \times \mathbf{k}\right) / R+\mathcal{O}\left(c^{-4}\right) .
$$

The only effect in $\delta \dot{x}_{p}$ to be accounted for here is the post-Newtonian gravitational deflection from the spherically symmetric part of the gravitational field of the Sun. The explicit formulas will be published elsewhere. The effect amounts to $10 \mu$ as for a source situated at a distance of $1 \mathrm{pc}$ and observed at the limb of the Sun. One can check that the effect is larger than $1 \mu$ as if $|\mathbf{X}| \leq 10 \mathrm{pc}$ and the source is observed within $2.3^{\circ}$ from the center of the Sun. If at least one of these conditions is violated one can put $\mathbf{k}=\sigma$.

\subsection{Parallax}

Now we have to get rid of the parallax (that is to transform $\mathbf{k}$ into a unit vector 1 directed from the barycenter of the solar system to the source):

$$
\mathbf{l}(t)=\mathbf{X}(T) /|\mathbf{X}(T)|
$$


Let the parallax of the source be defined as

$$
\pi(t)=1 \text { a.u. } /|\mathbf{X}(T)|,
$$

the parallactic parameter $\Pi$ be given by

$$
\Pi(t)=\pi(t) \mathbf{x}_{s}(t) / 1 \text { a.u., }
$$

and finally the observed parallactic shift of the source be defined as

$$
\boldsymbol{\pi}(t)=\mathbf{l}(t) \times(\Pi(t) \times \mathbf{l}(t)) .
$$

With these definitions to sufficient accuracy one has

$$
\mathbf{k}=-\mathbf{l}\left(1-|\pi|^{2} / 2\right)+\pi(1+1 \cdot \Pi)+\mathcal{O}\left(\pi^{3}\right) .
$$

The second-order effects in (11) proportional to $\pi^{2}$ are less than $3 \mu$ as if $|\mathbf{X}| \geq 1$ pc. The second-order terms can be safely neglected if $|\mathbf{X}| \geq 2 \mathrm{pc}$.

\subsection{Proper motion}

The last step of the algorithm is to provide a reasonable parametrization of the time dependence of 1 and $\pi$ caused by the motion of the source relative to the solar system barycenter. The following simple model for the coordinates of the source is adopted here

$$
\mathbf{X}(T)=\mathbf{X}_{0}+\mathbf{V} \Delta T+\mathcal{O}\left(\Delta T^{2}\right),
$$

where $\Delta T=T-T_{0}, \mathbf{X}_{0}=\mathbf{X}\left(T_{0}\right)$, and $\mathbf{V}$ is the BRS velocity of the source evaluated at the initial epoch $T_{0}$. This model allows one to consider single stars or components of gravitationally bounded systems, periods of which are much larger than the time span of observations. In more complicated cases special solutions for binary stars, etc. should be considered. Depending on the source and time span of the observations higher-order terms in (12) can also be considered. Substituting (12) into definitions of $l$ and $\pi$ one gets

$$
\begin{aligned}
\pi(t) & =\pi_{0}\left(1-\mathrm{l}_{0} \cdot \mathrm{V} / X_{0} \Delta T+\mathcal{O}\left(\Delta T^{2}\right)\right), \\
\mathbf{l} & =\mathrm{l}_{0}+\mathrm{l}_{0} \times\left(\mathrm{V} \times \mathrm{l}_{0}\right) / X_{0} \Delta T+\mathcal{O}\left(\Delta T^{2}\right),
\end{aligned}
$$

where $X_{0}=\left|\mathbf{X}_{0}\right|, \pi_{0}=\pi\left(t_{0}\right)=1$ a.u. $/ X_{0}$.

The signals emitted at moments $T_{0}$ and $T$ are received by the observer at moments $t_{0}$ and $t$, respectively. Corresponding moments of emission and reception are related by the equations

$$
c(t-T)=\left|\mathbf{x}_{s}(t)-\mathbf{X}(T)\right|
$$

and similar equation for $t_{0}$ and $T_{0}$. The relativistic effects in (15) can be shown to be negligible. Let us denote $\Delta t=t-t_{0}$ as the time span of observations corresponding to the time span of emission $\Delta T$. These two time intervals are related as

$$
\Delta t=\left(1+c^{-1} \mathbf{l}_{0} \cdot \mathrm{V}\right) \Delta T-c^{-1}\left[\mathbf{x}_{s}(t)-\mathbf{x}_{s}\left(t_{0}\right)\right] \cdot \mathbf{l}_{0}+\mathcal{O}\left(\Delta T^{2}\right) .
$$


The first term in (16) is linear with respect to $\Delta T$, the second term represents a quasi-periodic effect with an amplitude of about $500 \mathrm{~s}$, giving a quasi-periodic term in apparent proper motion of the source (see, Klioner and Kopeikin, 1992). Eq. (16) results from a Taylor expansion of (15). Which terms of such an expansion are important depends on many factors. For example, for a large time span of observations terms quadratic in $\Delta T$ may become important.

It is easy to see from (13) that time dependence of parallax $\pi$ can be used to determine radial velocity of the source. This question has been investigated in more detail in Dravins et al. (1999). The "true" tangential and radial components of barycentric velocity $\mathbf{V}$ of the source can be defined by

$$
\mathrm{V}_{\mathrm{tan}}=\mathrm{l}_{0} \times\left(\cdot \mathrm{V} \times \mathrm{l}_{0}\right), \quad V_{\mathrm{rad}}=\mathrm{l}_{0} \cdot \mathrm{V} .
$$

Eqs. (13)-(14) can be combined with (16) to get the time dependence of $l$ and $\pi$ as seen by the observer. Collecting terms linear with respect to $\Delta t$ we get the definition of apparent tangential velocity $\mathrm{V}_{\tan }^{\mathrm{ap}}$ as the linear term in $\mathrm{l}(t)$, and of apparent radial velocity $V_{\text {rad }}^{\text {ap }}$ as the linear term in $\pi(t)$ :

$$
\mathrm{V}_{\mathrm{tan}}^{\mathrm{ap}}=\mathrm{V}_{\mathrm{tan}}\left(1+c^{-1} V_{\mathrm{rad}}\right)^{-1}, \quad V_{\mathrm{rad}}^{\mathrm{ap}}=V_{\mathrm{rad}}\left(1+c^{-1} V_{\mathrm{rad}}\right)^{-1} .
$$

With these definition the simplest models for $\pi(t)$ and $\mathbf{l}(t)$ as seen by the observer read (the higher-order terms are neglected here):

$$
\begin{aligned}
\pi(t) & =\pi_{0}-\pi_{0}^{2}\left(V_{\mathrm{rad}}^{\mathrm{ap}} / 1 \text { a.e. }\right) \Delta t+\ldots, \\
\mathbf{l}(t) & =\mathrm{l}_{0}+\mu_{\mathrm{ap}} \Delta t+\mu_{\mathrm{ap}} c^{-1}\left(\left[\mathbf{x}_{s}(t)-\mathbf{x}_{s}\left(t_{0}\right)\right] \cdot \mathbf{l}_{0}\right)+\ldots \\
\mu_{\mathrm{ap}} & =\pi_{0}\left(\mathrm{~V}_{\mathrm{tan}}^{\mathrm{ap}} / 1 \text { a.e. }\right)
\end{aligned}
$$

Apparent proper motion is denoted $\boldsymbol{\mu}_{\mathrm{ap}}$ here. The factor $\left(1+c^{-1} V_{\mathrm{rad}}\right)^{-1}$ in (18) has been discussed in, e.g., Stumpff (1985) and Klioner and Kopeikin (1992). This factor may become very large and is one of the possible explanations of the well-known problem of superluminal motions in quasars and active nuclei of galaxies.

If both $\mathbf{V}_{\mathrm{tan}}^{\mathrm{ap}}$ and $V_{\mathrm{rad}}^{\mathrm{ap}}$ can be determined from observations one can immediately restore the "true" velocities $V_{\text {tan }}$ and $V_{\text {rad }}$. However, even if it is not the case $V_{\tan }^{\text {ap }}$ and $V_{\text {rad }}^{\text {ap }}$ are useful by themselves. Note that the radial velocities as measured by Doppler observations are affected by a number of factors not influencing positional observations and do not coincide with either $\mathrm{V}_{\mathrm{rad}}$ or $V_{\mathrm{rad}}^{\mathrm{ap}}$.

\section{References}

Brumberg, V.A., Klioner, S.A., Kopejkin, S.M., 1990, In: Inertial Coordinate System on the Sky, Lieske, J.H., Abalakin, V.K. (eds.), Kluwer, Dordrecht, 229-240.

Dravins, D., Lindegren, L., Madsen, S., 1999, Astron. Astrophys., 348, 10401051.

Klioner, S.A., 1991a, Sov. Astron., 35, 523-530. 
Klioner, S.A., (1991b, Relativistic Reduction of Astronomical Observations. PhD thesis, St. Petersburg State University (in Russian)

Klioner S.A., Kopeikin, S.M., 1992, Astron. J., 104, 897-914.

Stumpff, P., 1985, Astron. Astrophys, 144, 232-240. 